\title{
CARABIDAE (COLEOPTERA, INSECTA) NO CULTIVO DE TABACO EM SANTA CRUZ DO SUL, RS, BRASIL
}

\author{
Mylena Thaine Siqueira ${ }^{1}$ \\ Vinícius Ferreira ${ }^{2}$ \\ Cleder Pezzini ${ }^{3}$ \\ Andreas Köhler ${ }^{4}$
}

\begin{abstract}
RESUMO
A família Carabidae pertence à ordem Coleoptera, formada por besouros de hábito cursorial e predatório. A ocorrência de carabídeos pode determinar características importantes para o plantio de tabaco, além de contribuir para o controle biológico de insetos pragas. O objetivo do trabalho foi identificar as espécies de Carabidae amostradas em lavoura orgânica e convencional de tabaco e a sua distribuição, conforme o tipo da vegetação adjacente ao plantio. Foram realizadas coletas semanalmente durante três safras, de 2011 a 2014. As áreas adjacentes eram compostas por diferentes composições paisagísticas. Em cada linha de amostragem, foram determinados quatro pontos de coleta (pontos "fora", "borda", "dentro" e "meio"). As armadilhas utilizadas para a coleta foram do tipo Malaise e pit-fall. Foi amostrado um total de 1.820 indivíduos adultos de Carabidae, sendo 1.513 no cultivo orgânico e 307 em cultivo convencional de tabaco. No que se diz respeito aos espécimes, foram identificados sete táxons de Carabidae, sendo: Calosoma granulatum, Cicindela spp., Galerita spp., Lebia concinna, Megacephala (Tetracha) spp., Odontocheila spp. e Scarites spp. Através deste estudo, foi possível conhecer mais sobre a distribuição de Carabidae na cultura do tabaco, bem como o papel imprescindível da vegetação adjacente ao cultivo para a presença destes insetos.
\end{abstract}

Palavras-chave: Predadores. Controle biológico. Biodiversidade

\begin{abstract}
The family Carabidae belongs to the order Coleoptera, formed by beetles of cursorial and predatory habits. The incidence of carabids may determine important characteristics to tobacco planting, in addition to contribute to the biological control of pest insects. The study aimed to identify the species of Carabidae sampled in organic and conventional managed tobacco crops and their distribution, according to the type of vegetation adjacent to cultivating site. Samplings were conducted weekly for three crop seasons, from 2011 to 2014. The surrounding areas were composed of different landscape compositions. At each sampling line, four collection points were determined (in the "outside", "edge", "inside" and the "middle" of cultivating site). The traps used for the collect were the Malaise and Pit-fall. A total of 1,820 adults of Carabidae was sampled, wherein 1,513 in organic farming and 307 in conventional tobacco cultivation. As regards specimens, seven taxa of Carabidae were identified: Calosoma

\footnotetext{
1 Aluna do Ensino Médio na Escola Estadual de Ensino Médio Ernesto Alves de Oliveira. < $\underline{\text { mylena- }}$ siqueira@hotmail.com>

2 Aluno do Curso de Ciências Biológicas - Bacharelado da Universidade de Santa Cruz do Sul (UNISC). <vinicius--ferreira@outlook.com>

3 Biólogo e Mestrando em Fitotecnia na Universidade Federal do Rio Grande do Sul (UFRGS). <cleder.pezzini@hotmail.com>

${ }^{4}$ Professor do Departamento de Biologia e Farmácia na Universidade de Santa Cruz do Sul (UNISC). <andreas@unisc.br>
} 
granulatum, Cicindela spp., Galerita spp., Lebia concinna, Megacephala (Tetracha) spp.,Odontocheila spp. and Scarites spp. By this study, it was possible to establish more about the distribution of Carabidae in tobacco crops, as well as the essential role of vegetation adjacent to the culture for the presence of these insects.

Keywords: Predators. Biological control. Biodiversity

\section{INTRODUÇÃO}

O tabaco (Nicotiana tabacum L.) pertence à família das plantas solanáceas, sendo este cultivado para fins comerciais em mais de 125 países. O Brasil é o segundo maior produtor de tabaco atrás apenas da China, ocupando a duas décadas ininterruptas a liderança global quando o assunto é exportação. (LANDONI, 1990; GUERRERO, 1995; VENCATO et al., 2011).

A cultura do tabaco possui grande importância econômica, devido ao elevado valor comercial e à capacidade de empregar considerável número de pessoas, tanto no cultivo como na industrialização (SPECHT et al., 2006). Assim, estudos envolvendo essa cultura são de extrema importância por conta de sua elevada produtividade, principalmente na Região Sul do país.

Segundo Moraes (2014), muitos problemas são ocasionados em virtude das grandes áreas utilizadas na cultura do tabaco, por conta da grande demanda do uso de insumos externos destinados ao cultivo convencional. A modificação dos ecossistemas naturalmente diversificados, devido aos processos de produção, gera a simplificação da estrutura do ambiente, fragmentação do habitat e, principalmente, a redução da biodiversidade local.

Essa alteração de ambiente causa uma instabilidade ecológica que acarreta em problemas fitossanitários, relacionados, na maioria das vezes, ao aumento de populações de insetos-praga e a diminuição de inimigos naturais, uma vez que a regulação dos ecossistemas naturais, proporcionadas pela biodiversidade, são comprometidas ou perdidas (MORAES, 2014).

Em agroecossistemas, a biodiversidade é considerada a base dos sistemas de produção, onde estão contidas as plantas cultivadas, as plantas espontâneas, os animais, os micro-organismos e outros componentes bióticos (AGUIAR-MENEZES, 2004). A agricultura orgânica trata-se do manejo sustentável de agroecossistemas, com enfoque sistêmico, que privilegia a preservação ambiental, a biodiversidade, os ciclos biogeoquímicos e a qualidade de vida humana (RICCI et al., 2006). 
A classe Insecta é responsável por constituir o maior e mais diverso grupo entre todos os seres vivos. Tendo aproximadamente um milhão de espécies já identificadas, perfazem em torno de $70 \%$ de todos os animais existentes. Porém, de acordo com especialistas, esse número corresponde a apenas $20 \%$ do total de espécies a serem descobertas e identificadas. Os insetos ocupam os mais distintos nichos ecológicos, habitando vegetais, solo e águas, além de contribuírem a para as cadeias vitais do planeta (AZEVEDO FILHO \& PRATES JÚNIOR, 2005; GALLO et al., 2002; PARRA et al., 2002; TRIPLEHORN \& JONNSON, 2011).

A ordem Coleoptera abriga os insetos popularmente conhecidos por besouros. São considerados importantes agentes da riqueza da entomofauna por compreenderem aproximadamente $40 \%$ das espécies dentro da classe Insecta e $30 \%$ do reino Animalia. Possuem cerca de 360.000 espécies descritas, podendo ser encontradas em uma grande variedade de habitats e nichos ecológicos (TRIPLEHORN \& JOHNSON, 2011).

Coleoptera é dividida em quatro Subordens: Archostemata, Myxophaga, Adephaga e Polyphaga. Além disso, as duas últimas subordens, Adephaga e Polyphaga, são as maiores e apresentam representantes de importância agrícola, incluindo espécies predadoras e pragas. Adephaga possui besouros com hábitos ativos, sendo a maioria dos representantes predadores por apresentarem mandíbulas bem desenvolvidas. Dentre as famílias dessa subordem, Carabidae é a mais representativa, contendo espécies terrestres e predadoras (TRIPLEHORN \& JONNSON, 2011).

Segundo Moraes (2014), ainda são poucas as informações sobre a biodiversidade de Coleoptera na cultura do tabaco, sobretudo ao que se refere à abundância e à diversidade de insetos-praga e inimigos naturais. Além das espécies reconhecidas como pragas agrícolas, ocorre grande diversidade de insetos não nocivos e/ou benéficos à cultura do tabaco. Dentre eles, destacam-se os coleópteros predadores, pertencentes às famílias Carabidae, Staphylinidae e Coccinellidae.

Os indivíduos da família Carabidae são frequentemente encontrados sob pedras, troncos, folhas, casca de árvores e detritos (BORROR \& DELONG, 1969), onde grande parte das espécies, tanto os indivíduos adultos como as larvas, são predadores de outros artrópodes, porém, poucas espécies são ectoparasitas, detritívoras ou herbívoras (LÖVEI \& SUNDERLAND, 1996; MARINONI, 2001). Essa família é diversificada e abundante em agroecossistemas e os besouros destacam-se como inimigos naturais de outros insetos, considerados pragas de culturas agrícolas e plantios florestais. 
Coleópteros predadores, principalmente das famílias Carabidae e Staphylinidae, são importantes componentes em culturas agrícolas, uma vez que são intimamente ligados ao controle populacional de insetos-praga, alimentando-se geralmente de afídeos, ovos e larvas de crisomelídeos, curculionídeos e outros coleópteros e larvas de lepidópteros. Devido à sua capacidade de controle, podem ser utilizados com sucesso em programas de manejo integrado de pragas (MIP) por meio de aplicações de técnicas de controle biológico por conservação (KROMP, 1999; HOLLAND \& LUFF, 2000; MENEZES \& AQUINO, 2005).

Segundo os autores Holland \& Luff (2000), no intuito de aumentar a ocorrência das famílias predadoras para que estas possam atuar como agentes de controle biológico de pragas torna-se então indispensável à avaliação das influências das culturas e demais habitats naturais presentes nos ambientes de agroecossistemas.

Portanto, objetivou-se no presente trabalho identificar as espécies de Carabidae coletado em lavoura orgânica e convencional de tabaco e a sua distribuição, conforme o tipo da composição florística adjacente ao plantio.

\section{MATERIAIS E MÉTODOS}

O município de Santa Cruz do Sul (29 43’59"S, 52²4’52"O) localiza-se na encosta inferior do Nordeste do Estado do Rio Grande do Sul, a $155 \mathrm{~km}$ de Porto Alegre, na região da Depressão Central. Pertencendo à unidade Patamares da Serra Geral, esta unidade está associada a morros testemunhos que avançam sobre a depressão do rio Jacuí. A altitude varia entre 70 a 150 m acima do nível do mar; o clima é classificado como subtropical temperado, com temperatura média de $19^{\circ} \mathrm{C}$, com máxima de $42^{\circ} \mathrm{C}$ e mínima de $5^{\circ} \mathrm{C}$; as chuvas caem entre 100 e 126 dias ao ano, com precipitações de 1.300 a 1.800mm (LEIFHEIT, 1978; COLLISCHONN, 2001).

A pesquisa foi realizada em lavouras de tabaco com manejo orgânico e convencional, localizadas no Centro Mundial para o Desenvolvimento Agronômico, Extensão e Treinamento (ADET) na propriedade da empresa JTI (Japan Tobacco International), no município de Santa Cruz do Sul, RS, Brasil. A lavoura orgânica é inspecionada anualmente e recebe as seguintes certificações: CEE 2092/91 (União Europeia) e USA NOP - 7 CFR Part 205 (USA National Organic Program).

As coletas foram realizadas semanalmente durante três safras, entre os anos de 2011 e 2014, do início dos cultivos até a colheita final. Na safra 2011/2012, foram realizadas 14 coletas, já entre o período de 2012/2013, 10 coletas, e na última safra ocorrida em 2013/2014, 
09 coletas. Os pontos de coleta distribuíram-se de modo a formar linhas de amostragens, no sentido fora-dentro da lavoura.

As lavouras orgânicas possuíam diferentes composições na área adjacente ao cultivo, sendo que, na safra:

- 2011/2012, as Linhas 1 e 2 possuíam uma vegetação adjacente composta de mata nativa e arbustos; e na Linha 3, uma área agrícola abandonada.

- 2012/2013, a Linha 1 possuía uma área de adubação verde composta de sorgo; a Linha 2, com vegetação adjacente composta por plantas forrageiras de crescimento espontâneo; e na Linha 3 com uma vegetação composta por espécies arbustivas (campo sujo).

- 2013/2014, a Linha 1 possuía uma vegetação adjacente composta por plantas forrageiras de crescimento espontâneo; já a Linha 2 continha uma área de adubação verde composta de sorgo.

Na lavoura convencional, na safra 2011/2012, a vegetação adjacente era composta de mata nativa e, nas safras 2012/2013 e 2013/2014, com área de campo limpo (gramíneas).

Em cada linha de amostragem, foram determinados quatro pontos de coleta (pontos "fora", "borda", "dentro" e "meio"), separados por aproximadamente 8 m de distância (Figura 1). Em cada ponto de coleta, foi instalada uma armadilha de interceptação de voo do tipo Malaise e quatro armadilhas de queda do tipo pit-fall.

Após as coletas, o material foi encaminhado ao Laboratório de Entomologia da Universidade de Santa Cruz do Sul para triagem e identificação até nível taxonômico de ordem, conforme Azevedo Filho \& Prates Júnior (2005). Posteriormente a identificação, o material foi tombado e conservado em álcool 70\%. Os indivíduos pertencentes à família Carabidae foram identificados até o nível de gênero ou espécie através das bibliografias específicas sobre esta família. 
Figura 1 - Croqui demonstrando as lavouras de tabaco manejadas organicamente durante as safras e a distribuição das linhas (L1, L2 e L3).

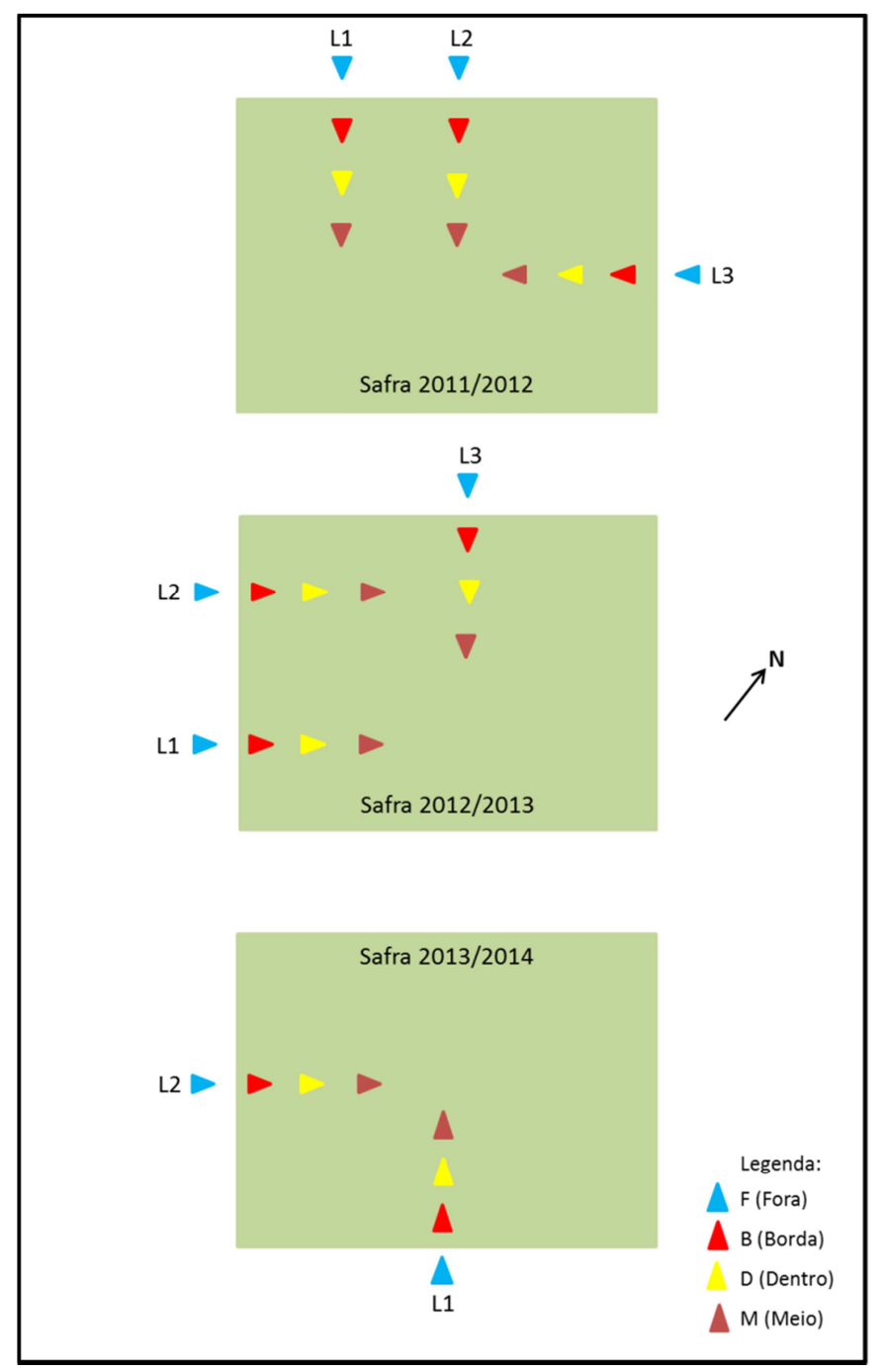

\subsection{Análise dos dados}

O cálculo da dominância dos táxons seguiu a fórmula $\mathbf{D}=(\mathbf{i} / \mathbf{t}) \mathbf{x 1 0 0}$, onde $\mathbf{D}=$ dominância (\%), $\mathbf{i}=$ total de indivíduos de um determinado táxon e $\mathbf{t}=$ total de indivíduos coletados, sendo que D > 10\% Eudominante (Ed), D 5 - 10\% Dominante (D), D 2 - 5\% Subdominante (Sd), D 1 - 2\% Recessiva (Rc) e D < 1\% Rara (Rr) (PALISSA et al., 1979).

A constância dos táxons coletados foi calculada através da fórmula $\mathbf{C}=\mathbf{p} \times 100 / \mathbf{N}$, onde $\mathbf{C}=$ constância $(\%), \boldsymbol{p}=$ número de coletas em que o táxon se fez presente, e $\boldsymbol{N}=$ número total de coletas efetuadas; sendo agrupadas como $\mathrm{C}>50 \%$ Constante $(\mathrm{C}), \mathrm{C} 25-$ $50 \%$ Acessória (As) e C < 25\% Acidental (Ac) (BODENHEIMER, 1955). 
Os índices de diversidade de Shannon e Equitabilidade J, foram obtidos através do programa Palaeontological Statistics (HAMMER et al., 2001).

\section{RESULTADOS E DISCUSSÃO}

Foi amostrado um total de 1.820 indivíduos adultos de Carabidae, sendo 1.513 no cultivo orgânico e 307 em cultivo convencional de tabaco. Na safra 2011/2012 foram coletados 539 indivíduos, na colheita seguinte, a de 2012/2013, 671 indivíduos, já na ultima safra em 2013/2014, 610 indivíduos coletados (Tabela 1).

Tabela 1 - Total de indivíduos amostrados em cada ponto ao longo das três safras

\begin{tabular}{llcccc}
\hline Safra & & Fora & Borda & Dentro & Meio \\
\hline 2011/2012 & & & & & \\
& Linha 1 & 74 & 44 & 14 & 15 \\
& Linha 2 & 85 & 20 & 32 & 17 \\
& Linha 3 & 20 & 16 & 18 & 13 \\
$2012 / 2013$ & Conv. & 114 & 26 & 21 & 10 \\
& & & & & \\
& Linha 1 & 63 & 57 & 39 & 82 \\
& Linha 2 & 82 & 32 & 29 & 12 \\
& Linha 3 & 120 & 25 & 29 & 19 \\
$2013 / 2014$ & Conv. & 16 & 30 & 20 & 16 \\
& Linha 1 & 100 & 32 & 43 & 79 \\
& Linha 2 & 172 & 62 & 39 & 29 \\
& Conv. & 33 & 10 & 01 & 10 \\
\hline Conv. - Convencional & & & &
\end{tabular}

Coombes \& Sotherton (1986) observaram em um estudo sobre a dispersão de adultos de besouros predadores terrestres das famílias Carabidae e Staphylinidae em culturas de cereais, dentro de cultivos e a partir de suas bordas, que os besouros podem ser coletados desde a borda até $200 \mathrm{~m}$ no interior dos cultivos.

Em relação aos espécimes coletados durante as três safras, foram identificados sete táxons de Carabidae, sendo Megacephala (Tetracha) spp. Hope, 1838 com 1.008 indivíduos (55\%); Lebia concinna Brulle, 1838 com 492 indivíduos (27\%); Odontocheila spp. Laporte, 1834 com 175 indivíduos (10\%); Scarites spp. Fabricius, 1775 com 90 indivíduos (5\%), Cicindela spp. Linné, 1758 com 34 indivíduos, Galerita spp. Fabricius, 1801, com 19 indivíduos, Calosoma granulatum Perty, 1830, com 02 indivíduos e os demais espécimes representaram $3 \%$ (Tabela 2 ). 
Tabela 2 - Total de indivíduos, Constância (Con) e Dominância (Dom) dos espécimes de Carabidae coletados em cada linha em cultivos Orgânico (Org.) e Convencional (Conv.) de tabaco ao longo das três safras.

\begin{tabular}{|c|c|c|c|c|c|c|c|c|c|c|c|c|c|c|c|c|c|c|c|c|c|c|c|c|}
\hline Safra 2011/2012 & \multicolumn{6}{|c|}{ Org. - Linha 01} & \multicolumn{6}{|c|}{ Org. - Linha 02} & \multicolumn{6}{|c|}{ Org. - Linha 03} & \multicolumn{6}{|c|}{ Conv. } \\
\hline Táxons & $\mathrm{F}$ & $\mathrm{B}$ & $\mathrm{D}$ & $\mathrm{M}$ & Con & Dom & $\mathrm{F}$ & $\mathrm{B}$ & $\mathrm{D}$ & $\mathrm{M}$ & Con & Dom & $\mathrm{F}$ & $\mathrm{B}$ & $\mathrm{D}$ & $\mathrm{M}$ & Con & Dom & $\mathrm{F}$ & $\mathrm{B}$ & $\mathrm{D}$ & $\mathrm{M}$ & Con & Dom \\
\hline Calosoma granulatum & 0 & 0 & 0 & 0 & - & - & 0 & 0 & 0 & 0 & - & - & 0 & 0 & 0 & 0 & - & - & 0 & 0 & 0 & 0 & - & - \\
\hline Cicindela spp. & 2 & 1 & 1 & 1 & Ac & $\mathrm{Sd}$ & 3 & 0 & 4 & 0 & Ac & $\mathrm{Sd}$ & 0 & 0 & 0 & 1 & Ac & $\mathrm{Rc}$ & 0 & 0 & 1 & 1 & Ac & $\mathrm{Rc}$ \\
\hline Galerita spp. & 0 & 0 & 1 & 0 & Ac & $\mathrm{Rr}$ & 2 & 0 & 0 & 1 & Ac & $\mathrm{Rc}$ & 2 & 1 & 1 & 5 & As & $\mathrm{Ed}$ & 1 & 1 & 2 & 0 & As & $\mathrm{Sd}$ \\
\hline Lebia concinna & 6 & 5 & 5 & 3 & $\mathrm{C}$ & Ed & 3 & 5 & 5 & 0 & $\mathrm{C}$ & $\mathrm{D}$ & 1 & 1 & 8 & 0 & $\mathrm{C}$ & $\mathrm{Ed}$ & 0 & 0 & 0 & 1 & Ac & $\mathrm{Rr}$ \\
\hline Megacephala spp. & 48 & 32 & 6 & 9 & $\mathrm{C}$ & $\mathrm{Ed}$ & 41 & 7 & 6 & 6 & $\mathrm{C}$ & Ed & 9 & 13 & 5 & 3 & $\mathrm{C}$ & $\mathrm{Ed}$ & 38 & 14 & 10 & 5 & $\mathrm{C}$ & $\mathrm{Ed}$ \\
\hline Odontocheila spp. & 11 & 2 & 0 & 1 & $\mathrm{C}$ & $\mathrm{D}$ & 28 & 2 & 9 & 7 & $\mathrm{C}$ & Ed & 0 & 1 & 2 & 0 & Ac & $\mathrm{Sd}$ & 63 & 11 & 8 & 1 & $\mathrm{C}$ & Ed \\
\hline Scarites spp. & 7 & 4 & 1 & 1 & $\mathrm{C}$ & $\mathrm{D}$ & 8 & 6 & 8 & 3 & $\mathrm{C}$ & $\mathrm{Ed}$ & 8 & 0 & 2 & 4 & $\mathrm{C}$ & $\mathrm{Ed}$ & 12 & 0 & 0 & 2 & Ac & $\mathrm{D}$ \\
\hline
\end{tabular}

\begin{tabular}{|c|c|c|c|c|c|c|c|c|c|c|c|c|c|c|c|c|c|c|c|c|c|c|c|c|}
\hline \multirow{2}{*}{$\begin{array}{l}\text { Safra 2012/2013 } \\
\text { Táxons }\end{array}$} & \multicolumn{6}{|c|}{ Org. - Linha 01} & \multicolumn{6}{|c|}{ Org. - Linha 02} & \multicolumn{6}{|c|}{ Org. - Linha 03} & \multicolumn{6}{|c|}{ Conv. } \\
\hline & $\mathrm{F}$ & $\mathrm{B}$ & $\mathrm{D}$ & $\mathrm{M}$ & Con & Dom & $\mathrm{F}$ & $\mathrm{B}$ & $\mathrm{D}$ & $\mathrm{M}$ & Con & Dom & $\mathrm{F}$ & $\mathrm{B}$ & $\mathrm{D}$ & $\mathrm{M}$ & Con & Dom & $\mathrm{F}$ & $\mathrm{B}$ & $\mathrm{D}$ & $\mathrm{M}$ & Con & Dom \\
\hline Calosoma granulatum & 0 & 0 & 0 & 0 & - & - & 1 & 0 & 0 & 0 & Ac & $\mathrm{Rr}$ & 0 & 0 & 0 & 0 & - & - & 0 & 0 & 0 & 0 & - & - \\
\hline Cicindela spp. & 0 & 1 & 4 & 1 & As & $\mathrm{Sd}$ & 0 & 3 & 0 & 1 & As & $\mathrm{Sd}$ & 2 & 1 & 2 & 2 & As & $\mathrm{Sd}$ & 0 & 1 & 0 & 0 & Ac & Ec \\
\hline Galerita spp. & 0 & 0 & 0 & 0 & - & - & 1 & 0 & 0 & 0 & Ac & $\mathrm{Rr}$ & 1 & 0 & 0 & 0 & Ac & $\mathrm{Rr}$ & 0 & 0 & 0 & 0 & - & - \\
\hline Lebia concinna & 11 & 9 & 12 & 30 & $\mathrm{C}$ & Ed & 47 & 8 & 13 & 2 & $\mathrm{C}$ & $\mathrm{Ed}$ & 108 & 13 & 16 & 6 & $\mathrm{C}$ & $\mathrm{Ed}$ & 4 & 4 & 5 & 4 & $\mathrm{C}$ & Ed \\
\hline Megacephala spp. & 47 & 44 & 22 & 46 & $\mathrm{C}$ & $\mathrm{Ed}$ & 29 & 21 & 11 & 6 & $\mathrm{C}$ & Ed & 3 & 10 & 10 & 9 & $\mathrm{C}$ & $\mathrm{Ed}$ & 12 & 24 & 14 & 12 & $\mathrm{C}$ & Ed \\
\hline Odontocheila spp. & 1 & 3 & 1 & 5 & $\mathrm{C}$ & $\mathrm{Sd}$ & 1 & 0 & 2 & 1 & As & $\mathrm{Sd}$ & 2 & 1 & 1 & 2 & $\mathrm{C}$ & $\mathrm{Sd}$ & 0 & 0 & 1 & 0 & Ac & $\mathrm{Rc}$ \\
\hline Scarites spp. & 4 & 0 & 0 & 0 & As & $\mathrm{Rc}$ & 3 & 0 & 3 & 2 & $\mathrm{C}$ & $\mathrm{D}$ & 4 & 0 & 0 & 0 & As & $\mathrm{Sd}$ & 0 & 1 & 0 & 0 & Ac & $\mathrm{Rc}$ \\
\hline
\end{tabular}

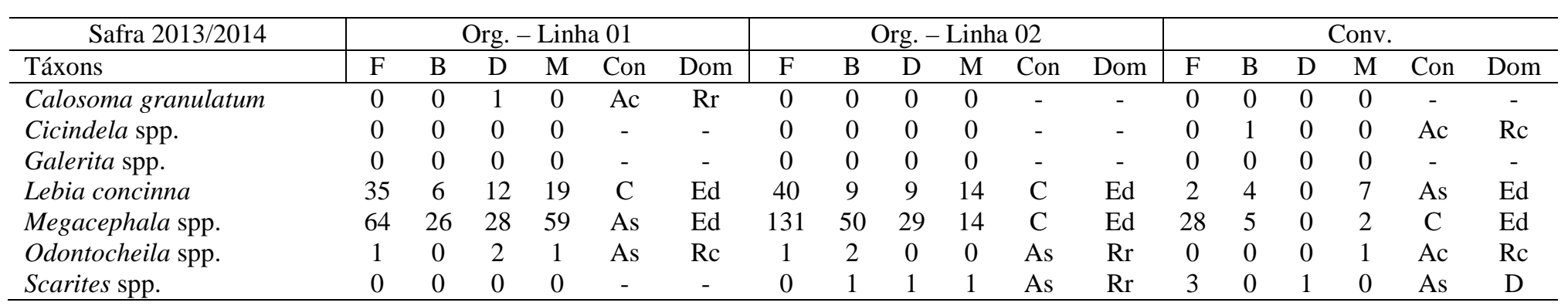

Scarites spp.

Pontos de coleta - F: Fora; B: Borda; D: Dentro; M: Meio.

Constância - C: Constante; As: Acessória; Ac: Acidental.

Dominância - Ed: Eudominante; D: Dominante; Sd: Subdominante; Rc: Recessiva; Rr: Rara. 
A vegetação adjacente as Linhas 1 e 2, no cultivo orgânico e no convencional da safra de 2011/2012, era composta de mata nativa, onde se observou a maior dominância dos gêneros Odontocheila e Scarites que estão associados a áreas com fragmentos florestais preservados. Os espécimes Megacephala (Tetracha) e Lebia concinna ocorreram tanto fora como dentro do cultivo, apresentando números decrescentes da borda para o centro do cultivo.

Cividanes \& Santos-Cividanes (2008) destacaram que Odontocheila nodicornis habita, preferencialmente, fragmentos de florestas, hábitats com microclima estável. Assim sendo, a presença de um fragmento florestal próximo dessas culturas também pode ter sido decisiva para a colonização desse predador.

A superdominância de $O$. nodicornis em cana-de-açúcar indica que a espécie encontrou nessa cultura, condição ambiental similar ao de fragmento florestal. A área de reflorestamento, com presença de plantas herbáceas, proporciona que carabídeos sejam mais diversificados e abundantes, podendo servir como refúgio durante as épocas de entressafra das culturas (CORREIA, 2013).

Segundo Martins, (2008); Correia, (2013); Vieira et al., (2008), a ocorrência de Scarites sp. associa-se com áreas florestais e/ou de reflorestamento adjacentes ao cultivo de soja e milho, o mesmo observado no tabaco.

Na safra 2012/2013, a vegetação adjacente as Linhas 1 e 2 eram compostas apenas por plantas forrageiras de crescimento espontâneo e na Linha 3 composto por pequenos arbustos, sem áreas com fragmentos florestais próximo. Somente as espécies Megacephala (Tetracha) e Lebia concinna foram consideradas dominantes ou eudominantes nos dois tipos de manejo (Tabela 3). Geralmente, outras espécies associadas a áreas com mata não foram representativas, nesta safra.

Martins et al. (2009) constatou que Megacephala (Tetracha) brasiliensis é uma espécie que também apresentou preferência por área agrícola, quando comparado com área de fragmento florestal adjacente.

Conforme Quinteiro et al. (2012), Megacephala (Tetracha) brasiliensis se fez presente quase que exclusivamente na área antropizada, caracterizando-se como espécie oportunista e podendo ser selecionada como indicadora de ambientes alterados.

Segundo Thomazini \& Thomazini, (2001) a ocorrência de Lebia concinna, juntamente com Callida sp. e Nabis sp., ocorre desde o período vegetativo da soja, aumentando gradativamente, no final do enchimento de grãos. Já no cultivo do tabaco, a população de L. concinna se manteve constante ao longo de toda a safra, não sofrendo grandes alterações no número de indivíduos amostrados ao longo das coletas. 
Os resultados da safra 2013/2014 corroboram com os dados da safra anterior, pois esta possuía as mesmas espécies dominantes, sendo que a vegetação adjacente era muito parecida com a da safra precedente (Tabela 2).

Dessa forma, a diversidade e a abundância de inimigos naturais nas culturas podem ser positivamente correlacionadas com a presença de fragmentos florestais e com a natureza da vegetação nas adjacências (KROMP, 1999). Esses componentes têm valor econômico e a presença dos mesmos deve ser mantida e incorporada aos agroecossistemas, tendo em vista que favorecem a ocorrência em alta densidade de insetos predadores (ASTERAKI et al., 1995; THOMAS et al., 1991) e colaboram para uma produção agrícola sustentável (ALTIERI et al., 2003). A redução, ou mesmo a ausência, desses habitats não agrícolas pode diminuir a ocorrência de predadores, limitando o potencial desses inimigos naturais em controlar as pragas (COOMBES \& SOTHERTON, 1986; THOMAS et al., 1991).

Os valores do índice de Shannon no cultivo orgânico foram maiores do que no convencional, apresentando uma maior diversidade. Somente no último ano, o índice é menor no cultivo orgânico devido ao baixo número de Megacephala (Tetracha) amostrado.

Se compararmos todas as linhas durante as safras estudadas, a equitabilidade fica entre 0,43 e 0,63 e somente as Linhas 2 e 3 da safra 2011/2012 se destacando com valores ao redor de 0,8 pois as espécies eudominantes não ultrapassam os 44,7\%. Já nas demais linhas esses valores chegam a 75\% de dominância de uma espécie (Tabela 3). Considerando também que a escala de equitabilidade vai de zero a um, é possível perceber que os índices são relativamente baixos em quase todos os pontos. Segundo Odum (1985), estes valores são normais em áreas antropizadas (agricultura) e de baixa biodiversidade.

Comparando os dois tipos de manejo do cultivo, observou-se uma maior densidade de Carabidae no cultivo orgânico em comparação ao convencional. Lee et al. (2001) e Landis et al. (2005) observaram que o uso de inseticidas na cultura do milho reduz a atividade e a densidade da comunidade de Carabidae, onde esses insetos, possivelmente, utilizam as áreas adjacentes aos cultivos como abrigo, durante os períodos de aplicação de inseticidas e colheitas. Com isso, a redução do número de aplicações de produtos químicos pode promover uma maior abundância e diversidade desses besouros (ELLSBURY et al., 1998). 
Tabela 3 - Número de táxons e de indivíduos; Índices de Diversidade de Shannon e Equitabilidade da fauna de Carabidae em cultivo orgânico e convencional de tabaco, nas safras 2011/2012 e 2012/2013 e 2013/2014.

\begin{tabular}{|c|c|c|c|c|}
\hline & \multicolumn{4}{|c|}{ Safra $2011 / 2012$} \\
\hline & L1 & L2 & L3 & Conv. \\
\hline Táxons & 6 & 6 & 6 & 6 \\
\hline Indivíduos & 147 & 154 & 67 & 171 \\
\hline Shannon & 1,134 & 1,449 & 1,442 & 1,093 \\
\hline \multirow[t]{3}{*}{ Equitabilidade $\mathbf{J}$} & 0,6329 & 0,8088 & 0,805 & 0,6099 \\
\hline & \multicolumn{4}{|c|}{ Safra 2012/2013 } \\
\hline & L1 & L2 & L3 & Conv. \\
\hline Táxons & 5 & 7 & 6 & 5 \\
\hline Indivíduos & 241 & 155 & 193 & 82 \\
\hline Shannon & 0,9157 & 1,128 & 0,8559 & 0,6988 \\
\hline \multirow[t]{3}{*}{ Equitabilidade $\mathbf{J}$} & 0,5689 & 0,5799 & 0,4777 & 0,4342 \\
\hline & \multicolumn{4}{|c|}{ Safra 2013/2014 } \\
\hline & L1 & $\mathrm{L} 2$ & Conv. & \\
\hline Táxons & 4 & 4 & 5 & \\
\hline Indivíduos & 254 & 302 & 54 & \\
\hline Shannon & 0,6962 & 0,6551 & 0,9644 & \\
\hline Equitabilidade $\mathbf{J}$ & 0,5022 & 0,4725 & 0,5992 & \\
\hline
\end{tabular}

\section{CONCLUSÃO}

Com este estudo foi possível conhecer mais sobre a ecologia e a distribuição de insetos, neste caso Carabidae, que estão associados à cultura do tabaco. Podendo ainda entender melhor sua importância dentro do cultivo, além do papel imprescindível da vegetação adjacente ao cultivo para a presença destes insetos predadores.

As espécies de Carabidae Megacephala sp. e L. concinna prevaleceram em áreas de cultivo de tabaco. Por sua vez, Odontocheila sp. e Scarites sp. predominaram em fragmentos florestais adjacentes ao plantio.

No cultivo orgânico é possível notar que há maior biodiversidade e riqueza de Carabidae, em relação ao cultivo convencional, devido ao seu manejo que não utiliza produtos químicos. 


\section{REFERÊNCIAS}

AGUIAR-MENEZES, E. L. Diversidade vegetal: uma estratégia para o manejo de pragas em sistemas sustentáveis de produção agrícola. Seropédica: Embrapa Agrobiologia, 2004.

ALTIERI, M. A.; SILVA, E. do N.; NICHOLLS, C. I. O papel da biodiversidade no manejo de pragas. Ribeirão Preto: Holos, 2003.

ASTERAKI, E. J.; HANKS, C. B.; CLEMENTS, R. O. The influence of different types of grassland field margin on carabid beetle (Coleoptera, Carabidae) communities. Agriculture, Ecosystems \& Environment, n. 3, v. 54, p. 195-202, jul. 1995.

AZEVEDO FILHO, W. S.; PRATES JÚNIOR, P. H. S. Técnicas de coleta \& identificação de insetos. 2 ed. Porto Alegre: EDIPUCRS, 2005. (Série Zoologia, 4)

BODENHEIMER, F. S. Précis d'écologie animale. Paris: Payot, 1955.

BORROR, D. J.; DELONG, D. M. Introdução ao estudo dos insetos. Rio de Janeiro: USAID, 1969.

CIVIDANES, F. J.; SANTOS-CIVIDANES, T. M. Distribuição de Carabidae e Staphylinidae em agroecossistemas. Pesquisa Agropecuária Brasileira, Brasília, n. 2, v. 43, p. 157-162, fev. 2008.

COLLISCHONN, E. O. Espaço Natural na Região do Vale do Rio Pardo. In: VOGT, O. P.; SILVEIRA, R. L. L. (Orgs.). Vale do Rio Pardo: (re) conhecendo a região. Santa Cruz do Sul: EDUNISC, 2001. p. 19-46.

COOMBES, D. S.; SOTHERTONS, N. W. The dispersal and distribution of polyphagous predatory Coleoptera in cereals. Annals of Applied Biology [online], n. 3, v. 108, p. 461-474, jun. 1986.

CORREIA, E. T. Diversidade e distribuição sazonal de Carabidae (Insecta, Coleoptera) em diferentes culturas. Jaboticabal, 2013. 63 p. Dissertação (Mestrado), Programa de PósGraduação em Agronomia, Universidade Estadual Paulista (UNESP), 2013.

ELLSBURY, M. M. et al. Diversity and dominant species of ground beetle assemblages (Coleoptera: Carabidae) in crop rotation and chemical input systems for the Northern Great Plains. Annals of the Entomological Society of America, n. 5, v. 91, p. 619-625, abr. 1998.

GALLO, D. et al. Entomologia agrícola. Piracicaba: FEALQ, 2002.

GUERRERO, R. C. El cultivo del tabaco. São José: EUNED, 1995.

HAMMER, O.; HARPER, D. A. T.; RYAN, P. D. PAST: Paleontological statistics software package for education and data analysis. Palaeontologia Electronica [online], n. 1, v. 4, p. 19, maio 2001.

HOLLAND, J. M.; LUFF, M. L. The effects of agricultural practices on Carabidae in temperate agroecosystems. Integrated Pest Management Reviews, Holanda, n. 2, v. 5, p. 109129, jan. 2000. 
KROMP, B. Carabid beetles in sustainable agriculture: a review on pest control efficacy, cultivation impacts and enhancement. Agriculture, Ecosystems and Environment, n. 1-3, v. 74, p. 187-228, jun. 1999.

LANDIS, D. A. et al. Manipulating plant resources to enhance beneficial arthropods in agricultural landscapes. Weed Science Society of America, n. 6, v. 53, p. 902-908, nov. 2005.

LANDONI, J. H. de. Nicotiana tabacum L. IPCS INCHEM, 1990. Disponível em: <http://www.inchem.org/documents/pims/plant/nicotab.htm>. Acesso em: 04 fev. 2015.

LEE, J. C.; MENALLED, F. D.; LANDIS, D. A. Refuge habitats modify impact of insecticide disturbance on carabid beetle communities. Journal of Applied Ecology, v. 38, p. 472-483, 2001.

LEIFHEIT, O. J. Introdução ao estudo do meio ambiente de Santa Cruz do Sul. Porto Alegre: Movimento, 1978.

LÖVEI, G. L.; SUNDERLAND, K. D. Ecology and behavior of ground beetles (Coleoptera: Carabidae). Annual Review of Entomology, v. 41, p. 231-256, jan. 1996.

MARINONI, R. C. et al. Hábitos alimentares em Coleoptera (Insecta). Ribeirão Preto: Holos, 2001.

MARTINS, I. C. F. Análise de fauna, flutuação populacional e preferência pelo habitat de Carabidae e Staphylinidae (Coleoptera) na região de Guaíra, Estado de São Paulo. Jaboticabal, 2008. 77 p. Dissertação (Mestrado), Programa de Pós-Graduação em Agronomia, Universidade Estadual Paulista Faculdade de Ciências Agrárias e Veterinárias, 2008.

MARTINS, I. C. F. et al. Análise de fauna e flutuação populacional de Carabidae e Staphylinidae (Coleoptera) em sistemas de plantio direto e convencional. Revista Brasileira de Entomologia, São Paulo, n. 3, v. 53, p. 432-443, 2009.

MENEZES, E. L. A.; AQUINO, A. M. Coleoptera terrestre e sua importância nos sistemas agropecuários. Seropédica: Embrapa Agrobiologia, 2005.

MORAES, J. Comunidades de Coleoptera em cultivos de tabaco (Nicotiana tabacum L.) em Santa Cruz do Sul, RS. Santa Maria, 2014. 136 p. Dissertação (Mestrado), Programa de PósGraduação em Agrobiologia, Universidade Federal de Santa Maria, 2014.

ODUM. E. P. Ecologia. 1. ed. Rio de Janeiro: Interamericana, 1985.

PALISSA, A.; WIEDENROTH, E. M.; KLIMT, K. Anleitung zum ökologischen Geländepraktikum. Potsdam: Wissenschaftliches Zentrum der Pädagogischen Hochschule Potsdam, 1979.

PARRA, J. R. P. et al. Controle Biológico no Brasil: parasitoides e predadores. São Paulo: Monole, 2002.

QUINTEIRO, N. T.; LOPES, J.; MARTINS, I. C. F. Diversidade de Carabidae (Coleoptera) amostrados em áreas de reflorestamento de mata ciliar e fragmento florestal, no Estado do Paraná. EntomoBrasilis [online], n. 3, v. 5, p. 217-222, set./dez. 2012.

RICCI, M. dos S. F. et al. Cultivo orgânico de cultivares de café a pleno sol e sombreado. Pesquisa Agropecuária Brasileira, Brasília, n. 4, v. 41, p. 569-575, abr. 2006. 
SPECHT, A. et al. Ocorrência de Rachiplusia nu (Guenée) (Lepidoptera: Noctuidae) em fumo (Nicotiana tabacum L.) no Rio Grande do Sul. Neotropical Entomology, Londrina, n. 5, v. 35, p. 705-706, set./out. 2006.

THOMAS, M. B.; WRATTEN, S. D.; SOTHERTON, N. W. Creation of "island" in farmland to manipulate populations of beneficial arthropods: predator densities and emigration. Journal of Applied Ecology [online], n. 3, v. 28, p. 906-917, dez. 1991.

THOMAZINI, M. J.; THOMAZINI, A. P. de B. W. Pragas e inimigos naturais associados à cultura da soja no Estado do Acre. Rio Branco, Embrapa Acre. Boletim de Pesquisa, 32, 2001. 22 p.

TRIPLEHORN, C. A.; JOHNSON, N. F. Estudo dos Insetos. São Paulo: Cengage Learning, 2011.

VENCATO, A. Z.; KIST, B. B.; SANTOS, C. et al. Anuário brasileiro do tabaco 2011. Santa Cruz do Sul: Gazeta Santa Cruz, 2011.

VIEIRA, L. et al. Comunidade de Carabidae (Coleoptera) em manchas florestais no Pantanal, Mato Grosso do Sul, Brasil. Iheringia, Série Zoologia, Porto Alegre, n. 3, v. 98, p. 317-324, set. 2008. 